\title{
El desafío territorial de la globalización. Reflexiones acerca del sistema regional chileno
}

Sergio Boisier

Instituto Latinoamericano y del Caribe de

Planificación Económica y Social (ILPES)

\section{Resumen}

En este artículo se analiza el desafío del desarrollo regional-local endógeno en Latinoamérica en el contexto de la dialéctica "territorialidad/globalización". Para ello se parte de la necesidad de comprender la globalización como un fenómeno multidimensional, reconociendolas dimensiones más frecuentemente consideradas en esto, como son la dimensión política, la económica, la social y una que no siempre es incluida de manera explícita, como lo es la dimensión geográfica. En relación con esta última se enfatiza la importancia de incluirla en términos de una dimensión geográfica interna, y también como una dimensión geográfica externa.

Comprender el desarrollo regional desde esta dialéctica "globalización/territorialidad" supone reconocer la interacción entre fuerzas internas y externas del desarrollo, que tienen la capacidad necesaria como para reconfigurar a las regiones. En este contexto, el desafío para los territorios es organizarse como regiones pivotales, que articulen una apertura externa económica 
con una apertura interna política, así como la interpenetración de lo público y lo privado en la gestión regional.

Las regiones pivotales son aquellos territorios con una organización compleja, en los cuales existen variedad de subsistemas jerarquizados de maneras no lineales. Las regiones pivotales también se caracterizan por su cultura generadora de identidad. Desde esta concepción orientada al desarrollo regional, la cultura regional se sintetiza como una manera de ver el mundo y de relacionarse con él. Estas regiones complejas también necesitan una modernización del Estado en términos territoriales, que en buena medida va de la mano de la capacidad para generar y organizar información.

\section{Globalización y reconfiguración territorial. Nueva epistemología regional y condiciones de éxito en un escenario globalizado}

La globalización, un nuevo mantra de la política económica contemporánea, tiene dimensiones políticas, económicas, sociales $\mathrm{y}$, últimamente aunque no menores, geográficas. Políticas en el sentido de contribuir a redibujar el "mapamundi" de la geografía política real, mediante el debilitamiento dela noción tradicional de Estado nacional y a través del paralelo fortalecimiento de los cuasi-Estados supranacionales y subnacionales; y políticas también, desde el punto de vista del poder, al consolidar la monopolaridad de la fuerza derivada de la hegemonía de los Estados Unidos de América. Económicas, al "mundializar" la producción manufacturera y el consumo por un lado, y al "mundializar" la competencia por mercados, tecnologías y capital. Sociales, en cuanto la globalización modifica la inserción laboral de grandes contingentes de población, pero sobre todo, debido a la "mundialización" de los mass media. Cable (1995) analiza los cinco mecanismos de la globalización: la integración tecnológica y económica, el capital financiero ("el fin de la geografía", según su terminología), las multinacionales y la inversión extranjera directa, el comercio de bienes y servicios, los movimientos de población, y la globalización de los regímenes de política para proveer bienes públicos internacionales. Cable también desmitifica varios aspectos del discurso de la globalización y examina las principales reacciones políticas a la globalización. Una cuestión poco examinada es el surgimiento de nuevas identidades, nuevas formas de nacionalismo (el nacionalismo "defensivo", el nacionalismo "positivo", la "nación competitiva", y el federalismo). Su gran conclusión es que el fenómeno de la globalización ha conducido a una reducción del espacio de maniobra de los gobiernos nacionales, en un número creciente de campos (pero no en todos), y ello no puede dejar de tener profundas implicaciones políticas.

Posiblemente la dimensión menos explorada, aunque no por esto menos radical en cuanto a la fuerza de los cambios que introduce, es la dimensión geográfica de la globalización, es decir, los cambios en la geografía política que se están produciendo en diversas escalas del mapa, desde el surgimiento de alrededor de veinte nuevos países en lo que va transcurrido de la década de los noventa, hasta la nueva geografía política en el interior de los países, que pugna por surgir por entre los crecientes resquebrajamientos de la rígida y obsoleta división política-administrativa, que no en pocos casos, tiene su origen en los Siglos XVII y XVIII.

Si la revolución científica y tecnológica introduce el cambio socio-económico a una velocidad exponencialmente creciente y en ámbitos también exponencialmente mayores, ¿por qué habrían de mantenerse sin daños las provincias, las regiones y los propios países, en cuanto divisiones territoriales?

Como lo señala Müller (1995):

"La participación internacional en el stock y en los flujos económicos de un país indica la intensidad de la globalización. Esta participación no se restringe a los procesos productivos y comerciales, financieros y de servicios; se extiende a la producción y la difusión de conocimiento y de tecnologías, a la gestión y a la cultura de un país. La globalización empuja a poblaciones e individuos, a países, regiones y localidades, a nuevas formas de interdependencia, en las cuales se experimentan formas inéditas de cooperación y de resolución de conflictos".

"Esta globalización competitiva acelera la desestructuración de organizaciones e instituciones locales $y$ regionales, nacionales y supranacionales, construidas en la postguerra, al tiempo que fuerza la emergencia de organizaciones e instituciones nuevas o reestructuradas, que no se asemejan nada a las anteriores, existentes hasta 
la presente ola internacionalizante de las economias nacionales".

De acuerdo con Boisier (1994), surgen dos escenarios nuevos que enmarcan, de ahora en adelante, los esfuerzos de intervención sobre el latente desarrollo regional o local endógeno: un nuevo escenario contextual un nuevo escenario estratégico para el desarrollo regional. Estos escenarios representan la síntesis de la dialéctica globalidad $y$ territorialidad, entre fuerzas exógenas y endógenas que se cruzan precisamente sobre los territorios organizados (no sobre cualquier tipo de partición territorial).

El escenario contextual del desarrollo regional queda configurado a su vez, por dos procesos. Por un lado, el proceso de apertura externa, de naturaleza esencialmente económica, resultado directo de la globalización y, en último término, de la microelectrónica, y por otro, el proceso de apertura interna, proceso de naturaleza política, expresión concreta de la fuerza de la descentralización, megatendencia de múltiples raíces.

El escenario estratégico del desarrollo regional queda configurado también por dos procesos. Uno, asociado a las nuevas modalidades de configuración regional (regiones pivotales, regiones asociativas y regiones virtuales) y otro, vinculado a la interpenetración entre lo público y lo privado, traducido en la aparición de modelos de gestión regional pública basados en criterios de gestión privados (regiones como cuasi-Estados y regiones como cuasi-empresas).

Es en estos escenarios, donde tiene lugar el "juego" de la globalización por parte de los territorios organizados (regiones, provinicias, comunas, etcétera). Para todo gobierno territorial, el desafío consiste en cómo ayudar al territorio de su jurisdicción a ocupar nichosquesean simultáneamente modernos, competitivos, equitativos y participativos.

Estos son los escenarios de la "modernidad". Para maximizar la posibilidad de salir del "juego" en condiciones "ganadoras", las regiones deben incorporar en su propia gestión, la flexibilidad, la velocidad, la maleabilidad y la imaginación creativa. La flexibilidad será para aumentar el abanico de respuestas a las demandas del entorno, tanto en términos de tamaño como de estructura, el concepto de "producción flexible" del post-fordismo tiene una reproducción analógica en el ámbito territorial. escenarios.

Los cuadros insertos a continuación dan cuenta de ambos
Cuadro 1

Escenario contextual del desarrollo regional descentralizado Apertura externa $\Leftrightarrow$ y apertura interna $(\Downarrow)$

(Globalización) + (Descentralización)

\begin{tabular}{|l|c|c|c|c|c|}
\hline \multirow{2}{*}{$\Rightarrow$} & & \multicolumn{2}{|c|}{ Alta } & \multicolumn{2}{c|}{ Baja } \\
\cline { 2 - 6 } & & Competitividad & Modernidad & Competitividad & Modernización \\
\hline Alta & Equidad & & & & \\
\hline Alta & Participación & & Chile 93 & & \\
\hline Baja & Equidad & Chile 93 & & & Chile 73 \\
\hline Baja & Participación & & & Chile 73 & \\
\hline
\end{tabular}

Nota: La ubicación de Chile en los casilleros es meramente referencial; pero muestra el importante cambio de ubicación del pás en el periodo considerado.

Cuadro 2

Escenario estratégico del desarrollo regional descentralizado

\begin{tabular}{||c|c|c|c||}
\hline Gestión $\Rightarrow$ & Cuasi-estados & Cuasi-empresas & $\begin{array}{c}\text { Ejemplos } \\
\text { colombianos }\end{array}$ \\
\hline Organización $\Downarrow$ & $\Uparrow$ & $\Downarrow$ & Antioquia, Valle \\
\hline Pivotales & $\Uparrow$ & $\Downarrow$ & $\begin{array}{c}\text { Nariño + Cauca } \\
\text { Valle + Choco }\end{array}$ \\
\hline Asociativas & Virtuales & $\Downarrow$ & $\begin{array}{c}\text { Antioquia/Cataluña } \\
\text { Valle/Lombardía } \\
\text { Occidente/Biobio }\end{array}$ \\
\hline
\end{tabular}

Nota: El sombreado indica là no existencia de esa situación.

La velocidad es una condición absolutamente indispensable para aprovechar las oportunidades (lo que constituye el verdadero secreto en el sistema de competencia), para entrar y salir rápidamente de acuerdos y de redes. La maleabilidad sirve para disponer de capacidad de adaptación estructural a los intersticios del entorno y a las características del propio medio regional (por ejemplo, en relación a la propia estructura de gobierno regional). La imaginación creativa es una condición esencial para un gobierno territorial reinventado, a la 
Osborne y Gaebler (1994), que permite "hacer más con menos", que permite movilizar los recursos psicosociales latentes en toda la comunidad, que complementan la parquedad usual de los recursos financieros, y que resultan ser, como es crecientemente reconocido, los elementos fundantes del desarrollo regional y local endógeno.

Desde el punto de vista estructural, cabe comentar acerca de las características de las regiones pivotales, consideradas como la organización territorial más apta para "competir" en los nuevos escenarios. En primer término, una región pivotal es, desde luego, un territorio organizado (los territorios, en general, recorren una gama de situaciones que incluyen el territorio "natural", el territorio "equipado" y el territorio "organizado") que es estructuralmente complejo. La "complejidad" es una condición necesaria para competir exitosamente en un escenario que es precisamente, complejo y en el cual las reglas lo son también. Es obvio que es difícil "ganar” en un juego complejo, para jugadores "simples". De aquí la necesidad de complejizar las estructuras regionales, como parte de los propios proyectos de desarrollo en cada región. Para expresarlo en términos más simples: si los productos que se configuran como las "estrellas" del comercio son productos con un alto contenido de conocimiento, no se puede pretender formar parte de ese conjunto intercambiando productos que sólo contienen información o muy escaso "conocimiento".

La complejidad hace referencia a:

1. La variedad de subsistemas que pueden reconocerse independientemente como formando parte del sistema (regional) completo;

2. La jerarquía o la capacidad decisional que muestran tales subsistemas;

3. La proporción de articulaciones no lineales presentes en el sistema, $\mathrm{y}$;

4. La recursividad presente en el sistema, esto es, la capacidad de sus elementos para ser simultáneamente causa y efecto (Morin, 1994).

En segundo término, una región pivotal es un territorio organizado que contiene y expresa una cultura, capaz a su vez, de generar una identidad y en consecuencia, capaz de solucionar virtuosamente la ecuación sociedad/territorio.

Cultura es la construcción social que sintetiza una cosmogonía, esto es, una manera de "mirar" el mundo, y una ética, es decir, de relacionarse con el mundo material y social. Más allá de la obviedad, más allá de reconocer que sin cultura productora de identidad no es posible siquiera emplear correctamente el vocablo región. La cultura e identidad se han convertido en factores de competitividad, al permitir introducir en bienes y servicios, un grado de diferenciación en un marco de competencia en el que las fuerzas homogeneizantes de la globalización tienen una presencia permanente.

\section{Las tendencias pesadas del crecimiento regional en Chile}

¿Qué está ocurriendo con el ordenamiento regional chileno? ¿Cuáles son las tendencias de crecimiento regional de largo plazo (las tendencias "pesadas") en Chile? ¿Cuáles son las regiones "ganadoras" y cuáles las "perdedoras” en los últimos decenios y qué implicaciones tiene esto desde el punto de vista de la globalización? ${ }^{1}$

Ahora están disponibles las cifras sobre la evolución del PIB regional para el periodo 1960-1992, debidamente homogeneizadas con respecto a los distintos cortes temporales con que hasta hace poco se presentaban. Los comentarios siguientes, que analizan tales cifras, se han extraído de un trabajo reciente de Boisier y Lira (1995).

La cantidad de información disponible, en términos absolutos y en términos relativos, es muy grande y hay que escapar a la tentación de efectuar una cantidad tal de procesamientos que la variedad de la información impida formar juicios certeros y rápidos. Por esta razón, en esta oportunidad se realizará un primer y sintético análisis de la información. Para ello se ocupa el software TAREA (versión 2.0) desarrollado en el ILPES, precisamente para este tipo de análisis; en particular se emplea la técnica conocida como "análisis diferencial/estructural", una técnica que permite diferenciar la importancia relativa de la composición sectorial del PIB regional y de la competitividad nacional de los sectores regionales, en la explicación de una dinámica económica de cada región que se muestra como mayor o

'Se ha puesto de moda hacer referencia a regiones "ganadoras" y "perdedoras" (Benko y Lipietz, 1992; Daher, 1994). Hay que tener cuidado con las sobresimplificaciones y con el reduccionismo, porque, a menos que se desee volver al equilibrio "casseliano", siempre y en cualquier periodo, hay regiones "ganadoras" y regiones "perdedoras". Lo importante es descubrir por qué razón algunas ganan y otras pierden. Véase, para un estudio más analítico: J.R., Cuadrado Roura (1994). 
como menor de la dinámica del pás en su conjunto en un mismo periodo de tiempo.

Deben mencionarse una serie de limitaciones tanto de la información como del propio método de análisis, limitaciones en general bien conocidas por los especialistas. La información se refiere a una desagregación de la cuenta del Producto por Sectores de Origen y la propia metodología de cálculo merece reparos; su desagregación por sectores de actividad económica regional es reducida y el análisis diferencial/estructural otorga un peso excesivo a la composición regional del PIB en el año inicial. Asimismo, el nivel de agregación de la información sólo permite hablar de las "tendencias pesadas", las cuales pueden ocultar y de hecho ocultan, "tendencias livianas" del cambio, que son muy importantes para provincias o para ramas productivas. El método se ubica en el campo de la "estática comparativa" y por tanto opera sólo con los puntos inicial y final de una serie, ocultando los cambios a lo largo de ella. Aún así, este tipo de estudio permite al analista regional "finamente entrenado", descubrir derroteros para investigaciones más acuciosas.

Como se ha dicho, nos adentramos rápidamente en un mundo en el cual la información y el conocimiento se convierten en el factor clave del desarrollo. En el capitalismo, la organización clave era la empresa; en el postcapitalismo, la organización clave, todavía no del todo configurada, es la organización capaz de procesar información y generar conocimiento. No está claro si tal papel lo cumplirá la universidad u otra forma organizacional. La apuesta en este sentido apunta más bien a relevar la importancia similar de varios "nodos" de la red de "inteligencia dispersa", que hoy tipifica la estructura de la producción de conocimiento, ya sea a nivel nacional, o a nivel regional.

De todos modos, es incuestionable la importancia, creciente por lo demás, del gobierno (regional y nacional en este caso) como "nodo" de dicha red, precisamente dentro de un modelo de política económica que apunta a un cambio radical en la forma de "hacer gobierno", privilegiando las modalidades indirectas de acción estatal (marcos regulatorios, equilibrios, información, etc.) por sobre el intervencionismo directo.

En otras oportunidades se ha llamado la atención, precisamente, sobre un nuevo papel de los gobiernos regionales que operan en marcos tipificados como economías de mercado, abiertas y descentralizadas. Se ha sostenido que el "reprocesamiento estructurado de la información", constituye una nueva tarea gubernamental, consistente en recoger la enorme y desordenada masa de información que circula "sobre" los agentes del desarrollo (empresarios, funcionarios, diri- gentes sociales, investigadores, etc.) con el objeto de "ordenarla" en función de un proyecto colectivo (de desarrollo) y devolverla a quienes toman las decisiones, reduciendo así el nivel de incertidumbre, los costos de transacción y generando un conjunto de decisiones individuales más a tono con el proyecto colectivo de desarrollo que la propia comunidad se da a sí misma.

En este sentido, todos los gobiernos regionales en Chile deberían estar trabajando en la interpretación fina de las cifras, cuyo análisis en forma agregada se mostrará a continuación. Para posicionarse adecuadamente en el difícil escenario del Siglo XXI, todo tomador de decisiones, en cualquier región, debe examinar el futuro a partir del conocimiento del pasado. Para cualquier inversionista potencial o empresario real, conocer el comportamiento macroeconómico de largo plazo de la región y disponer de, al menos, varias hipótesis que expliquen cuán bien o cuán mal lo ha hecho la región, es absolutamente fundamental a la hora de tomar decisiones que conlleven riesgos.

Hecho este breve preámbulo, ya es posible comentar algunos hechos sobresalientes. La primera conclusión que puede extraerse de la información, es que sólo las regiones I, II, IV, y RM (Tarapacá, Antofagasta, Coquimbo y Metropolitana) muestran un crecimiento mayor que el promedio del pás en el periodo de treinta años, $1960 / 1990$. Esto se explica porque todas estas regiones aumentaron su especialización en sectores de rápido crecimiento nacional entre 1960 y 1990 (pesca, electricidad, gas y agua, transporte y comunicaciones $y$, comercio ) y además, porque uno o varios de los sectores económicos de estas regiones se mostraron como más competitivos que iguales sectores de otras regiones. Estructura intersectorial y competitividad son los conceptos claves del éxito de estas cuatro regiones. La segunda, es que las regiones V, VI, VIII y IX, (Valparaíso, O'Higgins, Bíobío y Araucanía) son las regiones que muestran un peor resultado relativo. Todas ellas muestran un crecimiento menor que el promedio del país y en todos los casos, esto se explica por la combinación de un aumento de su especialización en sectores de lento crecimiento nacional, y por la menor competitividad de esos sectores en relación a iguales sectores ubicados en otras regiones. Las regiones de Valparaíso y del Bíobío en vez de atraer sectores modernos, han atraído actividades tradicionales. Hay que hacer notar que tanto la región de Valparaíso como la región del Bíobío (V y vII) son consideradas tradicionalmente como los contramagnetos de la Región Metropolitana. La tercera, es que las cinco regiones restantes (III, VII, y XI por un lado, -Atacama, Maule y Aysén- así como X y XII, por 
otro, - Los Lagos y Magallanes-, muestran resultados que responden a situaciones más complejas y diversificadas. Las regiones III, VII y XI anotan un crecimiento relativo mayor que el promedio del país. Ello resulta de combinar una temprana especialización en sectores de lento crecimiento nacional (se parecen entonces a las regiones V, VI, VIII y IX), pero que es más que compensada por una mayor competitividad de esos mismos sectores. Las regiones X $\mathbf{Y}$ XII han tenido un crecimiento relativo menor que el promedio del país, por el hecho de que a pesar de haber partido con una especialización adecuada en sectores de rápido crecimiento (se parecen a las exitosas regiones del Norte), esto fue contrarrestado por una competitividad sectorial menor, que en otras regiones que generan el mismo tipo de producción. No resulta aventurado atribuir a la distancia y a los costos asociados, parte de la explicación. La cuarta conclusión es que a partir de estos resultados, la geografía económica regional de Chile sugiere la aparición de algunos racimos o agrupaciones (clusters) supraregionales. Por ejemplo, la región de Tarapacá y la de Antofagasta podrían constituir una región asociativa, al igual que la región del Bíobío y la de la Araucanía también podrían configurar una región asociativa, a la luz de las más modernas concepciones del desarrollo regional. Pueden imaginarse ventajas en este tipo de asociación, para ambas regiones. Por ejemplo, la tradicional disputa entre Arica e Iquique tendería a diluirse en un espacio mayor y exitoso, en tanto que hay evidentes complementaridades de infraestructura y de otro tipo, entre Bíobío y la Araucanía, que facilitarían posiblemente la transformación de un mismo modelo de crecimiento lento a uno rápido.

La quinta gran conclusión es que las políticas de reconversión productiva y de fomento de la productividad se encuentran en el cruce de la cuestión regional en Chile, en particular con relación a la región de Valparaíso y a la región del Bíobío. En este último caso, la reconversión ha sido entendida erróneamente como una cuestión ligada sólo a la minería del carbón; en verdad y de acuerdo al nivel de agregación de la información, se trata de un problema de reconversión global de la región. Igual predicamento puede sostenerse en relación a la región de Valparaíso. Las dos acciones de largo plazo más importantes para los gobiernos de ambas regiones están vinculadas a preguntas como:

1. ¿qué sectores o ramas dinámicas del futuro deben ser estimuladas para localizarse en la región?
2. écómo mejorar ahora la productividad de las actuales actividades regionales? Como puede observarse, se trata de funciones completamente distintas de las tradicionales de la gestión pública.

Los dos gráficos siguientes muestran, el primero, una síntesis de las situaciones regionales y el segundo, ejemplos de políticas que deberían ponerse en ejecución en algunas regiones.

Cuadro 3

\section{Resumen de situaciones}

\begin{tabular}{|c|c|c||}
\hline $\begin{array}{c}\text { Ordenamiento territorial } \\
\text { chileno s/comportamiento } \\
\text { macroeconómico relativo } \\
\text { de las regiones 1960-1990 }\end{array}$ & $\begin{array}{c}\text { Aumento en productividad y } \\
\text { competitividad nacional de } \\
\text { sectores regionales }\end{array}$ & $\begin{array}{c}\text { Retroceso en productividad } \\
\text { competitividad nacional } \\
\text { de sectores regionales }\end{array}$ \\
\hline $\begin{array}{c}\text { Avance regional en la } \\
\text { especialización en sectores } \\
\text { dinámicos nacionales }\end{array}$ & $\begin{array}{c}\text { Regiones } \\
\text { I, II, IV, RM } \\
\text { (Regiones dinámicas) }\end{array}$ & $\begin{array}{c}\text { Regiones } \\
\text { X, XII }\end{array}$ \\
\hline $\begin{array}{c}\text { Retroceso regional en la } \\
\text { especialización en sectores } \\
\text { dinámicos nacionales }\end{array}$ & Regiones III, VII, XI & $\begin{array}{c}\text { Regiones V, VI, VII, IX } \\
\text { (Regiones rezagadas) }\end{array}$ \\
\hline
\end{tabular}

Cuadro 4

Objetivos básicos de políticas regionales según ciertas regiones

\begin{tabular}{||l|c|c||}
\hline & & Productividad global \\
\hline & & Región Los Lagos \\
& & Región Magallanes \\
\hline \multirow{2}{*}{ Reconversión global } & Región Atacama & Región Valparaíso \\
& Región Maule & Región Bíobío \\
& Región Aysen & Región Araucanía \\
\hline
\end{tabular}

En estas pocas observaciones sólo se ha explorado la rica información hasta ahora disponible acerca del movimiento de las regiones chilenas. La información muestra, simultáneamente, constancias y mudanzas 
en el escenario de las regiones chilenas. Al mismo tiempo son el reflejo de lo que el Estado y el mercado han hecho o han dejado de hacer; reflejan los equilibrios y los desequilibrios entre estas dos grandes fuerzas: la fuerza de la sociedad expresada unitariamente en su superestructura política y la fuerza atomizada de los agentes económicos. Cuando la información se desglosa por periodos, se puede apreciar que los cambios más profundos en las estructuras económicas regionales han sido provocados por la lógica de mercado, tan expresiva a partir de 1973.

Sería insensato desconocer los grandes méritos del paradigma vigente de la política económica y de la lógica de mercado, como su principal instrumento. Pero es preciso reconocer, al mismo tiempo, que la lógica de mercado no funciona con criterios territoriales, sino con criterios de rentabilidad microeconómica que producen efectos sobre el territorio de magnitud y direccionalidad, siempre discutibles desde un punto de vista social. El Estado tiene la obligación irrenunciable de hacer explícito el escenario territorial socialmente deseable, a fin de contrarrestar el puro resultado del mercado, más cerca de reproducir situaciones como la de Chiapas (en México) o la Santiago del Estero (en Argentina) que ordenamientos acordes con el superior interés nacional.

Estos resultados deben ser tenidos en cuenta a la hora de hacer un balance acerca del posicionamiento de las regiones chilenas en la globalización. Puede anticiparse, desde ya, que la situación que se observa en relación a las regiones del Bíobío y de Valparaíso no es nada promisoria en tal sentido.

\section{La modernización del Estado como condición para un buen posicionamiento en un mundo globalizado}

Las duras condiciones de la competencia internacional, que es sólo una parte del fenómeno de la globalización, obligan a modernizar la gestión de los territorios organizados (regiones), entendiendo por "modernización" la transformación de la región en una región inteligente, capaz de entender su propio entorno, capaz de acumular un conocimiento científico sobre ella misma (interno), capaz de interactuar con el entorno, capaz de anticipar el futuro mediante la construcción misma de él, capaz de aprender, capaz, en resumidas cuentas, de actuar en forma consonante y no disonante con la forma en que actúa el entorno regional (nacional e internacional).
Es difícil imaginar que el gobierno de una región en particular pudiese lograr el grado de modernización compatible con las necesidades de posicionamiento de su propia región, en tanto actúe por sí solo. Es por ello que puede sostenerse que la necesaria modernización de los gobiernos regionales hecha con el propósito de facilitar, nuevamente, un posicionamiento exitoso de sus propios territorios en el escenario internacional, requiere, como condición necesaria, la modernización del Estado, considerado este proceso desde un punto de vista territorial y regional.

En otro trabajo, Boisier (1995) ha desarrollado en profundidad este tema, configurando una "lista" de doce características que conformarían un Estado moderno, desde el punto de vista del funcionamiento del sistema regional.

\section{Cuadro 5}

Un Estado moderno desde el punto de vista territorial

1. Comprende y entiende su propia estructura sistémica territorial;

2. Es "inteligente", es decir, descentralizado y organizado en red;

3. Comprende la interrelación entre objetivos nacionales y el papel del territorio;

4. Puede en consecuencia, explicitar la contribución de cada región o territorio a cada objetivo;

5. A partir de ello, puede construir escenarios territoriales futuros;

6. Con tal información, incorpora la territorialidad en el proyecto político nacional;

7. Se reconoce a sí mismo como una institución desplegada en dos planos: el Estado nacional y un conjunto de cuasi-Estados regionales;

8. Acepta la variedad y por lo tanto permite y estimula la maleabilidad en las estructuras de gobierno y administración;

9. Incorpora en los cuasi-Estados regionales la velocidad y apoya la existencia de sistemas de información y análisis coyuntural;

10. Establece un marco regulatorio mínimo para facilitar la flexibilidad;

11. Estimula en las estructuras de los cuasi-Estados regionales el surgimiento de

la imaginación creativa y el despliegue de dos nuevas funciones (conducción política y animación social);

12. Se reconoce como un Estado "territorial" y es capaz de hacer conducción territorial tanto como conducción politica.

Para un lector familiarizado con la situación chilena, resulta fácil apreciar cuán lejos se está de configurar un Estado "moderno" desde 
el punto de vista territorial, independientemente de los serios esfuerzos en marcha. ${ }^{2}$

Dos de los aspectos que más pueden entrabar un adecuado posicionamiento de las regiones chilenas en el marco de la globalización radican, precisamente, en la falta de maleabilidad con relación a la estructura orgánica de los gobiernos regionales (un organigrama idéntico para las trece regiones, pasando por alto las enormes heterogeneidades entre ellas) y en la falta de flexibilidad de la configuración misma de las regiones, las que están definidas en número y en límites de forma autoritaria por un lado y al mismo tiempo, de una forma tan rígida, que se requiere jurídicamente y en forma exclusiva, de una iniciativa presidencial y de una ley de quórum calificado, para cualquier modificación. ${ }^{3}$

4. Oportunidades y desafíos para las regiones chilenas: ¿podrán aprovecharse las primeras y superarse los segundos?

El regreso de Chile al mundo democrático, en 1990, ha estado acompañado de un éxito macroeconómico e internacional sin precedentes en la historia del país. Clasificada ahora como una de las diez "economías emergentes" del mundo, Chile ha puesto nítidamente en práctica el concepto cepaliano del "regionalismo abierto". Miembro de la APEC, se apronta a pasar a ser el cuarto socio del NAFTA, se hacen los arreglos para ingresar al MERCOSUR y recientemente la Unión Europea ha ofrecido una asociación privilegiada a Chile. Además, el país multiplica los acuerdos bilaterales de variados tipos con países de distintos continentes.

Desde el punto de vista de la economía interna, los resultados son muy positivos en términos de crecimiento, inversión extranjera, control de la inflación, empleo, exportaciones, balanza de pagos, etc.

${ }^{2}$ Exactamente al escribir estas páginas, la prensa chilena ha dado cuenta de una importante reunión del Comité Interministerial para la Modernización de la Gestión Pública, organizada reunión del Comite lnterninisterial para la Modenización de la Gestion Publica, organizada para informar al Presidente de la Republica acerca de los avances en esta materia. Presidente, por su parte, ha reafirmado dos cuestiones basicas del proyecto modernizador mejorar la eficiencia del Estado (de la Administracion Publica) de forma que ella, al presta servicios al público, no atente a la dignidad de las personas y, avanzar en la descentralización en la toma de decisiones y en el manejo de los recursos. Véase reportaje del diario $L a$ Época 19/7/95, Santiago de Chile.

${ }^{3}$ Hay que comparar esta situación con la que se tiene en Colombia, en donde la Constitucion de 1991 (artículos 306 y 307), introduce los principios de autogeneración regional, de flexibilidad y de gradualidad.
Como sucede en otras partes, el éxito crea sus propios demonios: capital "golondrina", "narcocapital", sobrevaluación de la moneda nacional, incluso, surgimiento de alguna xenofobia trasnochada en contra de inversiones chilenas en el exterior.

Socialmente hay que distinguir entre la "situación social" del país y las "condiciones de vida de la población". En tanto la primera se muestra sin grandes conflictos (sin grandes disensos, pero también, sin grandes consensos), las condiciones de vida de la población acusan una distribución del ingreso extremadamente desigual. Según un último informe del Banco Mundial, los dos deciles superiores retienen $60 \%$ del ingreso, en tanto que los dos deciles inferiores capturan sólo cerca de $4 \%$. Sin embargo, debe reconocerse que las políticas y los programas sociales del gobierno están bien concebidos y muestran un grado apreciable de eficacia.

En resumen, el país enfrenta un escenario extremadamente favorable para acelerar su camino al desarrollo. La peculiar configuración geográfica del país ha generado una variedad de ecosistemas de Norte a Sur, dotando a casi todas las regiones de recursos naturales y de ventajas comparativas (estáticas) considerables, que en un contexto de intensa apertura externa, permite a muchas regiones realizar una "acumulación primaria" que debiera ser puesta al servicio de una complejización creciente de sus propias estructuras, a fin de acoplarse a la cabeza del cometa de la globalización y no a la cola. Hasta ahí las oportunidades.

Examinando las disparidades regionales y la competitividad territorial en la Comunidad Europea, J. R. Cuadrado (1994) anota las siguientes condiciones del éxito competitivo de varias regiones europeas, que necesariamente habría que reproducir acá:

1. "Evidentemente, algunas regiones tienen ciertas ventajas iniciales por aspectos locacionales ... para sacar provecho de ellas y ser competitivas, estas regiones deben tener una adecuada, si no avanzada, infraestructura de comunicaciones...i

2. El sistema urbano también juega un importante papel. Una gran ciudad bien equipada, con una imagen favorable y un sistema adecuado de núcleos urbanos complementarios, forma una base especialmente favorable para el desarrollo. 
3. La disponibilidadde recursos bumanos con una oferta estable de mano de obra calificada y una base educativa de alto nivel es una característica común a todas las regiones ganadoras.

4. La presencia de grandes empresas en sectores regresivos ba actuado en casi todos los casos como factor de repulsión para el intercambio comercial, mientras que el predominio de pequeñas empresas en una gran variedad de actividades se postula como un factor positivo.

\section{Otra clave es la accesibilidad de la región.}

6. La disponibilidad de productores de servicios avanzados.

7. La existencia de formas de gobierno regionales con autonomía frente a las instancias estatales y un sistema de cooperación entre las diferentes instanciasde gobierno $y$ las instituciones civiles.

8. Por último, la atractividad relativa de una región para atraer inversiones extranjeras o de activar las inversiones locales también tiene relación con el clima de cooperación social".

¿Cuántas de las trece regiones chilenas reunen todos o algunos de los requisitos de éxito competitivo? La respuesta a esta pregunta -que por obvia no requiere ser elaborada - es decepcionante.

Las cifras de la evolución del PIB regional comentadas más atrás, pero sobre todo, el conocimiento que se produce mediante su procesamiento, dan lugar a otras interrogantes no menos preocupantes. A comienzos de la década de los sesenta, las dos regiones (con excepción de la región Metropolitana) más complejas, utilizando las categorías de E. Morin (1994) citadas anteriormente, eran la región del Bíobío y la región de Valparaíso, precisamente las dos ubicadas en el peor de los mundos, después de treinta años: perdiendo competitividad, productividad y especialización en actividades dinámicas. ${ }^{4}$ En

${ }^{4}$ Es cierto que estos dos negativos procesos se han medido tomando como patrón de referencia al país en su conjunto. Si el patrón de referencia fuese el mundo, o sea precisamente el escenario globalizado, el resultado sería todavía peor. vez de aumentar el nivel de complejidad, que con toda seguridad acompaña a una mayor presencia regional de sectores dinámicos; se ha reducido la complejidad, a lo menos desde este punto de vista. En otras palabras, ambas regiones han perdido funcionalidad en relación a la globalización, aún cuando su comercio internacional sea mayor $o$ incluso creciente.

A la inversa, no resulta extraño que la región Metropolitana aparezca, en el mismo análisis, en un lugar privilegiado en el casillero ganador. De hecho, esta región acumula la mayor cantidad de los factores que Cuadrado apunta como condicionantes del éxito competitivo, y por ello no sorprenden en lo absoluto algunas de las conclusiones empíricas de un reciente estudio de Carlos de Mattos (1995), aunque difícilmente se puede concordar con sus conclusiones o inferencias más sustantivas, como por ejemplo, sostener que: "carece de toda viabilidad cualquier intento de configurar otra aglomeración de esta naturaleza". El error radica en visualizar la articulación región/nación/mundo (la globalización) sólo a través del prisma del tamaño, olvidando que la cuestión está en la complejidad que permite usar una suerte de "variedad necesaria" para un adecuado acoplamiento.

Quizás sea oportuno rescatar el trabajo de Vapñarsky (1969) sobre morfología de los sistemas urbanos nacionales (los arreglos conocidos como "rango-tamaño" y "primal") como resultante de los atributos sistémicos, cerramiento e interdependencia. A la luz de las proposiciones de Vapñarsky, la situación y la dinámica de la ciudad de Santiago de Chile no debiera sorprender en absoluto si Chile se considera un "sistema" de bajo cerramiento y baja interdependencia.

La productividad de la mano de obra representa un importante factor de competitividad, sobre todo en condiciones de apreciación sistemática de la moneda nacional, como es el caso de Chile en lo que va corrido de la década de los noventa. Un trabajo muy reciente de Uribe-Echevarría (1995), muestra cifras indicativas de una reducción de la productividad de la mano de obra en varias regiones, entre ellas, en la del Bíobío y en la de Valparaíso, para el periodo 1981/1989. Esto constituye un elemento adicional que dificulta un adecuado posicionamiento global de dos de las regiones más importantes de Chile.

Todavía se puede agregar otra cuestión, que llega a ser muy preocupante con respecto al futuro global del país y, por cierto, con respecto al tema de la inserción regional en la globalización. Esta cuestión se refiere al patrón sectorial y regional de la inversión en general y de la inversión privada internacional en particular. 
De acuerdo a Silva (1994), el catastro de proyectos no estatales para el periodo 1992-1996 preparado por la Corporación de Bienes de Capital, indica que $24.2 \%$ de las intenciones de inversión se dirige al sector minería, $20.0 \%$ al sector de industria y servicios, $16.5 \%$ a energía y combustibles, en tanto que $14.7 \%$ está constituído por proyectos del sector telecomunicaciones y transportes, $11.6 \%$ por proyectos forestales y $9.7 \%$ por proyectos turísticos. En el mismo trabajo se señala que el ordenamiento regional de estos proyectos muestra a la región de Magallanes concentrando 15.4\%, la del Bíobío $11.6 \%$, la de Antofagasta 10.3\%, la Metropolitana 9.5\%, la de Tarapacá 8.9\%, la de Atacama 5.6\%, la de Coquimbo $4.2 \%$ y la de la Araucanía $4.1 \%$. Se puede apreciar que la zona norte del país sigue mostrando muy buenas perspectivas de crecimiento, ya que las cuatro primeras regiones concentran casi el 30\% de la inversión revelada por este catastro. ${ }^{5}$

Aumenta la preocupación en medios académicos y políticos acerca de una suerte de "fatalidad económica" de Chile, bien representada por las cifras anteriores y otras que podrían agregarse. Es tal el nivel de las ventajas comparativas estáticas del país en sectores como minería, silvicultura, pesca y fruticultura (más o menos en el mismo orden), que se hace muy difícil modificar la lógica elemental del capital transnacional, que, con toda razón dentro de tal lógica, fluye a Chile, no para desarrollar novedosas actividades industriales/ manufactureras con tecnologías de punta, sino para aprovechar las condiciones ecosistémicas que generan las ventajas comentadas. ${ }^{6}$

Si el país va a pasar efectivamente a lo que se ha denominado como la "segunda fase del modelo exportador", ello requerirá una acción deliberada del Estado para ayudar a crear ventajas comparativas dinámicas o competitivas.

Si las regiones, o al menos algunas de ellas, van a posicionarse en forma adecuada en la globalización, más allá de un posicionamiento meramente comercial, por conveniente que ello sea en el corto plazo, habrá que desarrollar esfuerzos cooperativos (sector público/sector privado) para incorporar conocimiento, inicialmente, a los mismos

${ }^{5}$ Esto es perfectamente congruente con los resultados a los que llegan Boisier y Lira (1995) en el trabajo El subdesarrollo comienza al Sur.

${ }^{6}$ El día 18/7/95 los Senadores Sergio Bitar y Carlos Ominami, ingeniero y ex-Minitro de Minería (Gob. Allende) el primero economista y ex-Ministro de Economía (Gob. de Aylwin) el segundo, ofrecieron una conferencia de prensa para llamar la atención precisamente sobre esta cuestión.

productos ahora exportados con poca incorporación de progreso técnico.?

Al estudiar la apertura y evolución exportadora de las industrias dinámicas y su relación con el territorio, surge un asunto central desde el punto de vista del tema acá discutido, Riffo, Silva y de Mattos (1995) llegan a conclusiones o a sugerencias como las siguientes:

"En cuanto a la territorialidad, debe destacarse que los sectores dinámicos de apertura mayor que el promedio, con una participación igual o mayor al $10 \%$ de las ventas externas de este grupo, se concentran claramente en la región Metropolitana. Para tres de ellos (farmacéuticos y medicamentos, productos de cobre-3722-y 3833), esta región representa más del $80 \%$ de la ocupación y para cuatro de ellos los anteriores más imprentas y editoriales-3420) más del $80 \%$ del valor agregado. El más significativo de estos sectores en términos de ocupación es el 3420 que representa el 14\% de la ocuapción de los sectores dinámicos en 1992 y el $17 \%$ de esta misma categoría en la R.M.

Otras regiones en que se concentran estos sectores, de mayor apertura o participación en las ventas externas, son la V, VI, VII y puntualmente, la I; aunque para el conjunto de las dinámicas, la VIII Región tiene también alguna representatividad. Todas ellas muy lejos de la participación de la Región Metropolitana, como se ba analizado en otras partes de este trabajo.

Puede concluirse que los sectores de mayor vinculación al mercado externo dentro de los de mayor dinamismo productivo, están más concentrados en la Región Metropolitana, que el conjunto de las actividades dinámicas..."

${ }^{7}$ Hay que remarcar que no se piensa en forma simplista, que todas las regiones deba pasar de la producción de manzanas a la chips de silicona (aunque en el largo plazo ello puede ser una necesidad). Un quintal de trigo exportado para consumo es externa y formalmente idéntico a otro que contiene trigo manipulado genéticamente para ser utilizado como semilla. El contenido de conocimiento entre uno y otro es abismal, tal como lo es entre dos lingotes de cobre, uno producido por termometalurgia tradicional y otro por lixiviación bacteriana. 
Una cuestión adicional, que agrega preocupaciones en este campo, es observar la escasa iniciativa que las regiones (incluyendo bajo esta denominación tanto a sus órganos de gobierno y administración como a las organizaciones de la sociedad civil) muestran con respecto a la cuestión de la competitividad regional. Esto marca un agudo contraste, por ejemplo, con la situación colombiana, país en el cual el gobierno ha establecido la Consejería Presidencial para el Desarrollo y la Competitividad, con un órgano colegiado, el Consejo Nacional y con los correspondientes organismos regionales. Más aún, ha sido una iniciativa regional (en particular, del Departamento del Valle del Cauca) iniciar estudios sistemáticos en esta materia, contratando para ello la asesoría de MONITOR, la consultora de M. Porter. Puede agregarse que estas iniciativas se han originado en la Fundación para el Desarrollo Integral del Valle del Cauca (FDI), una institución imbricada en la Cámara de Comercio de Cali, (Millán; 1994).

Nada de esto parece acontecer en Chile, a pesar de que en algunas regiones existen las organizaciones empresariales que ya hubiesen podido tomar en sus manos este tipo de iniciativas. Sin perjuicio de la validez genérica de la afirmación precedente, hay que rescatar algunos pronunciamientos estratégicos recientes, por ejemplo, en la región de Valparaíso y con menos fuerza, en la del Bíobío.

La Estrategia Regional de Desarrollo de 1995 de la Región de Valparaíso (1995:50), al analizar las fortalezas y debilidades regionales apunta como la primera de las debilidades de "alta relevancia", la baja internacionalización de la economía regional y la escasa inversión extranjera, en tanto que la propuesta preliminar de 1994 de la Estrategia Regional de Desarrollo de la Región del Bíobío, define a la política de fomento de exportaciones como uno de los lineamientos globales de la estrategia para lograr el crecimiento económico y empleo permanente (1994:43).

La misma diferencia que existe entre un enchufe eléctrico y un puerto de computadora de más de veinte conexiones, es la que hay entre la inserción internacional del Siglo XX y la del Siglo XXI. No es que esta última sea más complicada, la cuestión es que es más ¡compleja!

\section{Bibliografía}

Benko, G., Lipietz, A., (1994) (ed), Las regiones que ganan. Distritosy redes. Los nuevos paradigmas de la geografía económica, Valencia, Edicions Alfons El Magnànim.

Boisier S. (1992), "La gestión de las regiones en el nuevo orden internacional: cuasi-Estados y cuasi-empresas", en: Estudio Sociales, núm. 72, C.P.U., Santiago de Chile.

(1994), "Postmodernismo territorial y globalización: regiones pivotales y regiones virtuales", en: Ciudad y Territorio. Estudios Territoriales, vol. II, núm. 102, M.A.P, Madrid.

- (1995), “La modernización del Estado. Una mirada desde las regiones”, en: Estudios Sociales núm. 85, C.P.U., Santiago de Chile.

- y L. Lira (1995), El subdesarrollo comienza al Sur, Santiago de Chile, ILPES/DPPR, Doc. 95/12, Serie Investigación.

Cable V. (1995), "What Future for the State?", Daedalus Journal of the American Academy of Arts and Sciences, Spring 1005.

Cuadrado Roura J. R. (1994), "Regional Disparities and Territorial Competition in the EC", en: J.R. Cuadrado, P. Nijkamp y P. Salvá (eds.) Moving Frontiers: Economic Restructuring, Regional Development and Emerging Networks, Avebury.

Daher, A. (1994), "Competencia: regiones ganadoras y perdedoras en Chile", Revista de Estudios Urbano Regionales, EURE, Instituto deEstudios Urbanos, Universidad Católica de Chile, Santiago de Chile.

de Mattos, C. (1995), Santiago de Chile 1975-1995, ¿Una nueva dinámica metropolitana en el escenario de la reestructuración y la globalización?, Instituto de Estudios Urbanos, Universidad Católica de Chile. 
Millán, F. (1994), Competitividad internacional de regiones, ILPES/DPPR, Doc. 94/22, Serie Ensayos, Santiago de Chile.

Morin, E. (1994), Introducción al pensamiento complejo, GEDISA Editorial, Barcelona, España.

Müller, G. (1995), O Desenvolvimento Regional-Local e os Sistemas Produtivos no Contexto da Globalizaşao, Descentralizaçao e Competitividade, Proposta de Pesquisa, DPR/IGCE-UNESP, Rio Claro, Sao Paulo, Brasil.

Osborne, D. y T. Gaebler (1994), Reinventando o Governo, MHC, Comunicaçao, Brasilia.

Región de Valparaíso/ Gobierno Regional (1995), Estrategia Regional de Desarrollo, Valparaíso, Chile.

Región del Bíobío/Intendencia Regional (1994), EstrategiaRegional de Desarrollo, Versión Preliminar, Concepción, Chile.

Riffo, L., V. Silva y C. de Mattos (1995), "Las tendencias locacionales de la industria en el marco de los procesos de reestructuración y globalización en Chile", Documento presentado al Seminario Internacional sobre Impactos Territoriales de los Procesos de Reestructuración Productiva, IEU, Universidad Católica de Chile, Santiago de Chile, julio de 1995.

Silva I. (1994), Inversión y crecimiento regional en Chile 1970-1990, Santiago de Chile, ILPES, Dirección de Proyectos y programación de Inversiones,

Uribe-Echevarría F. (1995), "Reestructuración económica y desigualdades inter-regionales en Chile", Documento presentado al Seminario Internacional sobre Impactos Territoriales de los Procesos de Reestructuración, Santiago de Chile, Instituto de Estudios Urbanos, Universidad Católica de Chile, julio de 1995.

Vapñarsky C. (1969), “On Rank-Size Distribution of Cities: An Ecological Approach", Economic Development and Cultural Change, Chicago, vol. 17, núm. 4. 


\section{Los Autores}

Sergio Boisier es Master of Arts in Regional Science de la Universidad de Pennsylvania. Ha sido Director a.i. de la Oficina de la CEPAL en Brasil (década de los 70) y Director Adjunto del Programa de Asesoría del ILPES (década de los 80) de las Naciones Unidas. Actualmente es Director de la Dirección de Políticas y Planificación Regionales del ILPES. Entre sus últimas publicaciones se destacan: " $\mathrm{La}$ modernización del Estado: una mirada desde las regiones", Globalización y Gestión del Desarrollo Regional, J. Medina, Cali, 1996. "Postscriptum sobre desarrollo regional. Modelos reales y modelos mentales", EURE, No 72, Santiago de Chile, 1998. "En busca do esquivo desenvolvimento regional: entre a caixa preta e o projeto político", Planejamento e Políticas Públicas, No 13, 1996, Brasil. "Posmodernismo territorial y globalización: regiones pivotales y regiones virtuales", Ciudad y Territorio y Estudios Territoriales, No 102, 1994, Madrid.

Carlos A. de Mattos es Profesor de Economía Regional y Urbana de los cursos de Magister del Instituto de Estudios Urbanos de la Pontificia Universidad Católica de Chile desde 1990 y Coordinador General de la Red Iberoamericana de Investigadores sobre Globalización y Territorio desde 1994. Miembro dell ILPES de las Naciones Unidas entre 1968 y 1989. Sus publicaciones más recientes son: "Modelos de crecimiento económico endógeno y divergencia interregional, ¿nuevos caminos para la gestión regional?", en: Bert Helmsing y Joao Guimaraes, Locality, State and Development. Essays in the Honour of Jos G. M. Hilhorst. Institute of Social Studies, La Haya, 1997. "Desenvolvimento sustentável nos territorios da globalizaçao. Alternativa de supervivência ou nova utopía?", en: Bertha Becker e Mariana Miranda (Eds), $A$ Geografia Politica do Desenvolvimento Sustentável. Editora UFRJ, Río de Janeiro, 1997 y 
compilador/editor conjuntamente con Hiernuax y Restrepo: Globalización y Territorio. Impactos y Perspectivas. F.C.E., Santiago de Chile, 1998.

Jean-Marc Fontan posee un doctorado en Antropología de la Universidad de Montréal y es profesor en el departamento de sociología de la Universidad de Québec en Montréal. Sus temas de investigación son "la globalización, los movimientos sociales y el desarrollo local". Entre sus publicaciones se cuentan Développement économique local: la théorie, la pratique, les expériences. (con D. G. Tremblay) Québec, Presses de l'Université du Québec, 1994, «Le projet Angus: une experience novatrice de mobilisation locale au coeur de Montréal» (con C. Yaccarini), Économie et solidarités, Vol. 28, Num. 1, 1996, pp. 65-81, y *Mouvements sociaux et communautarisation du tissu social: l'enjeu du XXIe sièclex, en: Klein, J.L., Tremblay, P.A. y H. Dionne. (dir) Au-delà du néoliberalisme: quel rôle pour les mouvements sociaux? Québec, Presses de 1'Université du Québec, 1997, pp. 197-210.

Daniel Hiernaux es doctor en Estudios de las Sociedades Latinoamericanas (orientación geografía, ordenamiento y urbanismo) por la Universidad de Paris III La Sorbona Nueva. Desde 1984, se desempeña como profesor investigador de la Universidad Autónoma Metropolitana Unidad Xochimilco, donde dirige actualmente el área de "sociedad y territorio" del Doctorado en Ciencias Sociales. Sus líneas de investigación son: "las políticas regionales y el impacto de la globalización en el territorio"; "la ciudad de México en la globalización y en particular la evolución de las periferias"; también "el turismo en el sistema mundial actual". Es editor y autor de varios, entre los cuales se destaca Nueva periferia, vieja metrópoli, el Valle de Chalco, ciudad de México, ed. UAM, 1996. Prepara en la actualidad un libro titulado Mexico, tendances et enjeux pour l'an 2000 para la Editorial l'Harmattan de París. Tecnología, sociedad y territorio y La geografía como metáfora de la libertad, textos de Eliseo Reclus se encuentran en curso de publicación por el Centro de Investigaciones Científicas Jorge L. Tamayo AC.

Juan-Luis Klein posee un doctorado en Geografía de la Universidad Laval y es profesor en el departamento de geografía de la Universidad de Québec en Montréal. Sus temas de investigación corresponden a "las comunidades territoriales", "la reconversión territorial", "los impactos territoriales de las nuevas tecnologías de comunicación" y 
"los efectos locales de la mundialización". Entre sus trabajos recientes, se puede consultar L'education géographique. Formation du citoyen et conscience territoriale (con S. Laurin) Québec, Presses de l'Université du Québec, 1998, Au-delà du néoliberalisme: quel rôle pour les mouvements sociaux? (con Tremblay, P.A. y H. Dionne) Québec, Presses de l'Université du Québec, 1997, y \&L'espace local àl'heure de la globalisation: la part de la mobilisation sociale», Cabiers de géographie du Québec, Vol. 41 Num. 114, 1997, pp: 367-377.

Peter Karl Kresl es profesor del Departamento de Economía de la Universidad de Bucknell, Lewisburg (Estados Unidos) desde 1969. Obtuvo su Ph. D en la Universidad de Texas en 1970. Es autor de Urban Economy and Regional Trade Liberalization (Praeger, 1992); The Impact of Free Trade on Canadian-American Border Cities (Univ. of Maine, 1993); The Competitiveness of Cities (OECD, 1995). Es editor de: Seen from the South (BYU Press, 1989); y North American Cities and the Global Economy (Sage, 1995). Su interés de investigación actual se centra en los siguientes temas: "ciudades y economía global", "cultura política" e "integración económica en Norteamérica y Europa Occidental". Ha sido Presidente de la Association for Canadian Studies en los Estados Unidos (1985-87) y Director Ejecutivo del International Council for Canadian Studies (1987-88). Ha sido profesor visitante en The Norwegian School of Economics, de la Universidad de Lund y también en la Universidad de Vermont.

Alicia Lindón es doctora en Ciencias Sociales con especialidad en Sociología por El Colegio de México. Es investigadora de El Colegio Mexiquense y profesora de la Universidad Iberoamericana. Sus líneas de investigación son: "los modos de vida urbanos y la cultura", "sociología de la vida cotidiana", "el enfoque biográfico y la intersubjetividad". Entre sus publicaciones se cuentan: De la trama de la cotidianeidad a los modos de vida urbanos. El Valle de Cbalco, El Colegio de México-El Colegio Mexiquense, en prensa. "El trabajo y la vida cotidiana. Un enfoque desde los espacios de vida", Economía, Sociedad y Territorio, 1997, $\mathrm{N}^{\circ} 1$, pp. 176-197. "El espacio y el territorio: contexto de significado en las obras de Simmel, Heidegger y Ortega y Gasset", Estudios Sociológicos, 1996, 40, pp. 129-141. Actualmente trabaja en la coordinación de una publicación colectiva sobre La vida cotidiana y su espacio-temporalidad.

Diane-Gabrielle Tremblay posee un doctorado en Ciencias Económicas de la Universidad de París I y es profesora en el departamento 
de economía y de gestión de la Tele-Universidad de la Universidad de Québec. También es presidente de la Asociación de Economía Política (Montréal). Sus temas de investigación son: "la economía del trabajo", "la innovación", "las políticas de desarrollo de la mano de obra" y "la asociatividad público-privada". Entre sus publicaciones se cuentan Développement économique local: la théorie, la pratique, les expériences. (con J.M. Fontan) Québec, Presses de 1'Université du Québec, Innovation, technologie et qualification. Multidimensionet complexitédu processus d'innovation, Québec, Presses de l'Univesité du Québec, 1996, y «Capital socioterritorial et milieux innovateurs» (con J.L. Klein, J.M. Fontan y S. Rousseau) en: Côté, S., Proulx, M.U. Espaces en mutation, Rimouski, GRIDEQ, 1998, pp. 119-133.

\section{INVITACIÓN}

Se invita a todos los investigadores interesados a participar con artículos referentes a los temas de la Revista. Para tal efecto se les invita a enviar sus colaboraciones bajo los siguientes lineamientos:

La entrega de un trabajo para su publicación en la revista Economía, Sociedad y Territorio implica para el colaborador el compromiso de no someterlo a otras publicaciones en español al momento de su aprobación para aparecer dentro de la revista. La publicación admite textos en otros idiomas encargándose ésta de la traducción al español. En los casos en los cuales sean enviados en español se anexará un ejemplar en el idioma original.

Se sugiere que los artículos tengan una extensión de 15 cuartillas como mínimo y 40 como máximo, incluidas gráficas, cuadros, notas y bibliografía; las reseñas podrán tener entre 5 y 10 cuartillas; cada número de la revista esta compuesto de 280 cuartillas como máximo de extensión (incluyendo cuadros, gráficas y todo material gráfico que se incluya en el número).

La presentación de los textos deberá enmarcar dentro de los siguientes lineamientos:

1.-Los artículos deberán presentarse en su versión final y completas ya que no se admitirán cambios iniciado el proceso de producción de la publicación.

2.-Los textos se presentarán impresos en matriz de puntos o en láser a doble espacio $(1,5)$ en tipo courier o times de 11 puntos; los textos que sean entregados mecanografiados deberán estar a doble espacio en cuartillas de 28 líneas y aproximadamente 64 golpes por línea sobre papel grueso; por ningún motivo serán recibidos sobre papel copia y/o copias al carbón. A estos materiales se le sumará el texto del artículo en procesador de palabras, de preferencia en un disquette de 3.5" y en Word perfect 5.1, Word 6 o almacenarse bajo código de TEXTO DOS (ASCII); en el caso de incluir cuadros, tablas, gráficas o mapas, se presentarán en archivos separados señalando únicamente 
la posible ubicación de éstos en el texto (no incluirlos en los archivos de texto).

3.-Las referencias bibliògráficas se harán en el texto, entre paréntesis, de acuerdo al siguiente modelo: apellido del autor(a), año de edición, número de la página.

Las notas de pie de página, los cuadros y las gráficas se agruparán al final del artículo después de la bibliografía. Las gráficas y cuadros deberán presentarse impresos en originales claros y precisos que permitan su publicación; en el caso de ser enviados en disquette deberán de estar almacenados en Excel 5 de ninguna manera serán recibidos en otro programa o versiones posteriores a ésta.

En el texto deberá de indicarse la colocación aproximadamente de estos materiales gráficos.

La lista de referencia bibliográficas deberán presentarse completas y en el siguiente orden: apellido y nombre del autor(a), año de edición, nombre del libro, país o ciudad, editorial. Ejemplo:

Legorreta, Jorge (1992), "Expansión urbana de la ciudad de México" en A. Bassols, J. Delgadillo y F. Torres (comps.), El desarrollo regional en México: Teoría y práctica, México, IIE, UNAM.

4.-La página inicial de cada artículo deberá contener: a) resumen en español con extensión máxima de 200 palabras; b) nombre del autor(a), su filiación institucional, grados académicos, área de interés principal y dos referencias bibliográficas de su autoría; c) número telefónico (fax), dirección electrónica (E-mail) y dirección postal para su eventual localización.

5.-Los artículos y su manejo son competencia del coordinador responsable del número al cual los autores tendrán que dirigirse para cualquier duda o aclaración.

\section{El Colegio Mexiquense, A.C.}
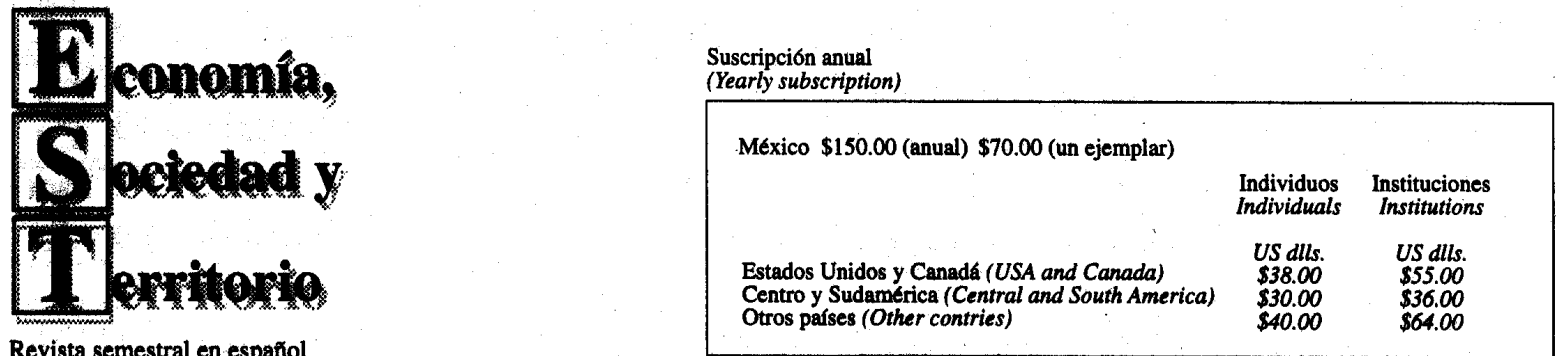

Revista semestral en espano

(A journal published twiece a year in Spanish)

Adjunto cheque o giro bancario a nombre de El Colegio Mexiquense, A.C., núm. (Enclosed bank check or order payable to)

Banco

Enviar a: El Colegio Mexiquense, A.C.

(Send to) Departamento de adquisiciones y libreria.

Apartado postal 48-D

Toluca 50120, Mexico

México

Nombre

(Name)

Dirección

CP

(Zip code) $\quad$ (City)

Pars

Teléfono $y$ fax

(Telephone and) 